\title{
Self-diffusion of supercritical water in extremely low-density region
}

$\operatorname{AUTHOR}(S)$ :

Yoshida, K; Matubayasi, N; Nakahara, M

\section{CITATION:}

Yoshida, K ...[et al]. Self-diffusion of supercritical water in extremely lowdensity region. JOURNAL OF CHEMICAL PHYSICS 2006, 125(7): 074307.

\section{ISSUE DATE:}

2006-08-21

URL:

http://hdl.handle.net/2433/50369

\section{RIGHT:}

Copyright 2006 American Institute of Physics. This article may be downloaded for personal use only. Any other use requires prior permission of the author and the American Institute of Physics. 


\title{
Self-diffusion of supercritical water in extremely low-density region
}

\author{
Ken Yoshida, Nobuyuki Matubayasi, and Masaru Nakahara ${ }^{a)}$ \\ Institute for Chemical Research, Kyoto University, Uji, Kyoto 611-0011, Japan
}

(Received 19 May 2006; accepted 13 July 2006; published online 18 August 2006)

\begin{abstract}
The self-diffusion coefficient $D$ for super- and subcritical water is determined by using the proton pulsed-field-gradient spin echo method at high temperatures and low densities. The density of water is ranged in the steamlike region from 0.0046 to $0.0650 \mathrm{~g} \mathrm{~cm}^{-3}$ at a supercritical temperature of $400{ }^{\circ} \mathrm{C}$, also at $0.0046-0.0079$ and $0.0046-0.0462 \mathrm{~g} \mathrm{~cm}^{-3}$ (the steam-branch densities on the coexistence curve and lower) at 200 and $300{ }^{\circ} \mathrm{C}$, respectively. The density is precisely determined on the basis of the PVT dependence of the proton chemical shift. The density-diffusivity products in the zero-density limit divided by the square root of the temperature, $(\rho D)^{0} / \sqrt{T}$, are $1.03,1.28$, and $1.44 \mathrm{fg} \mathrm{m}^{-1} \mathrm{~s}^{-1} \mathrm{~K}^{-1 / 2}$ (f, femto) at 200,300 , and $400{ }^{\circ} \mathrm{C}$, respectively. The $(\rho D)^{0} / \sqrt{T}$ obtained decreases with decreasing temperature and is significantly smaller than the temperature-independent value from the hard sphere model, $1.95 \mathrm{fg} \mathrm{m}^{-1} \mathrm{~s}^{-1} \mathrm{~K}^{-1 / 2}$. The marked temperature dependence reflects the presence of the strong attractive interaction between a pair of water molecules. The magnitude of the experimental $D$ values and the temperature dependence are well reproduced by the molecular dynamics simulation using TIP4P-FQ model. The initial slope of the product $\rho D / \sqrt{T}$ against $\rho$ is almost zero at $400{ }^{\circ} \mathrm{C}$ and slightly negative at $300{ }^{\circ} \mathrm{C}$. (C) 2006 American Institute of Physics. [DOI: 10.1063/1.2333511]
\end{abstract}

\section{INTRODUCTION}

Recently, we have developed a high-sensitivity multinuclear magnetic resonance (NMR) probe applicable for diffusion measurements at high temperatures and succeeded in determining in high precision the self-diffusion coefficient for sub- and supercritical water. ${ }^{1}$ Here we focus on the extremely low, gaslike density region, $\rho$ $=0.0046-0.0650 \mathrm{~g} \mathrm{~cm}^{-3}$ (corresponding to the pressure of 1.4-15.2 $\mathrm{MPa}$ at $400{ }^{\circ} \mathrm{C}$ ). It is interesting to test the simple gas kinetic theory by investigating how the self-diffusion of supercritical water depends on the presence of hydrogen bonding interaction at low densities. It has long been a fundamental issue how to describe the transport properties of gases and liquids from a molecular viewpoint. ${ }^{2-4}$ Our new experimental results will allow us to get a new insight into the low-density dynamics controlled by strong attractive intermolecular interactions. A molecular picture of the translational diffusion of reactive species including water itself is needed for the understanding of supercritical water reactions that have recently advanced a great deal. ${ }^{5-15}$ The temperatures and densities studied are also typical of steam in power plant and the transport properties in it are important for improving power generation efficiency. ${ }^{16,17}$

Previously, the self-diffusion coefficient $D$ for supercritical water has been obtained at a density of $0.07 \mathrm{~g} \mathrm{~cm}^{-3}$ (3.9 $\mathrm{M} ; \mathrm{M}=\mathrm{mol} \mathrm{dm}^{-3}$ ) and higher. ${ }^{1,18}$ It has been observed that $D$ increases steeply with decreasing density as the hydrogen bonds are broken. The steep increase has been explained by the approximate constancy of the product of the density and the self-diffusion coefficient, $\rho D$, on the basis of

\footnotetext{
a) Author to whom correspondence should be addressed. Electronic mail: nakahara@scl.kyoto-u.ac.jp
}

the Boltzmann equation applied for dilute gases. The dilutegas approximation, however, can be reliable only when the average intermolecular distance $\left(\rho_{n}^{-1 / 3} ; \rho_{n}\right.$, the number density) is significantly larger than the molecular size. Thus, it is desired to study supercritical water at much lower densities than before from a dynamical viewpoint. The lowest density investigated in this study is $0.0046 \mathrm{~g} \mathrm{~cm}^{-3}(0.26 \mathrm{M})$, which is an order of magnitude smaller than the previous. Our objective is (1) to determine the self-diffusion coefficient $D$ for supercritical water at sufficiently low densities where $D$ can be safely extrapolated to the zero-density limit, (2) to test the self-diffusion coefficient predicted by the kinetic theory for dilute gases at sufficiently low densities where the transport properties are mainly controlled by uncorrelated binary interactions, (3) to compare the experimental results with those obtained by molecular dynamics (MD) simulation performed at much lower densities than before, (4) to examine whether the influence of the attractive part of the potential is present or not when the temperature is varied over a wide range of $200-400{ }^{\circ} \mathrm{C}$, and (5) to examine how $D$ approaches the zero-density limit when the density is continuously lowered.

Water is of great interest because the attractive potential effect on the self-diffusion is particularly strong; the spherically averaged depth of the Lennard-Jones (LJ) potential well for water, $\varepsilon / k_{B}$, is $809.1 \mathrm{~K} .{ }^{19}$ In this study, the temperature scaled by the LJ potential well depth, $T^{*}=k_{B} T / \varepsilon$, is widely ranged from 0.58 to 0.83 . Thus we can scrutinize how the product $\rho D / \sqrt{T}$, the temperature-independent value in the hard sphere model, is changed with temperature. At a lower temperature of $200{ }^{\circ} \mathrm{C}(473 \mathrm{~K})$, in particular, the effect of attractive interactions on steam is expected to be large because the temperature is rather "low" when it is scaled by the potential well depth, $k_{B} T / \varepsilon=0.58$. It should be noted that the 
attractive hydrogen bonding interaction is remarkable even at a higher temperature of $400{ }^{\circ} \mathrm{C}$; one or two hydrogen bonds per water molecule persist in the vicinity of the critical density of $\rho_{c}=0.322 \mathrm{~g} \mathrm{~cm}^{-3} \cdot{ }^{20,21}$ Thus, the temperature dependence of the product $\rho D / \sqrt{T}$ in the zero-density limit is a good probe for the effect of attractive interaction on the selfdiffusion. The self-diffusion for low-density fluid has been intensively examined by Peereboom et al. for xenon. ${ }^{22}$ They determined the self-diffusion coefficient for xenon using the ${ }^{129} \mathrm{Xe}$ and ${ }^{131} \mathrm{Xe}$ pulsed-field-gradient spin echo (PGSE) method down to the reduced density $\rho_{r}=\rho / \rho_{c}=0.05$. It has been seen that the temperature dependence of $D$ for xenon is somewhat stronger than the hard sphere behavior, although a large experimental uncertainty is noted because of the narrow range of temperature at high values of $T^{*}=1.18-1.48$ $\left(\varepsilon / k_{B}\right.$ for xenon is $\left.231.0 \mathrm{~K}\right)$.

Self-diffusion coefficients have been determined for various supercritical fluids and gases by the van der Waals laboratory group, ${ }^{22-24}$ Kobayashi and co-workers, ${ }^{25,26}$ Jonas and co-workers, ${ }^{18,27}$ and Asahi and Nakamura ${ }^{28,29}$ using the PGSE method. Peereboom et al. found that the initial slope of $\rho D$ for xenon is almost zero, in contrast to the prediction of the strongly negative slope by the Enskog theory for dense gases. $^{2,3}$ The reports on the initial slope of $\rho D$ against $\rho$ for other substances are limited by experimental difficulties. The initial slope has been reported to be almost zero or slightly negative for substances with a weaker attractive potential than that of water by using PGSE or tracer methods; methane $\left[\varepsilon / k_{B}=148.6 \mathrm{~K} ; T^{*}=1.15-2.07, \rho_{r} \geqslant 0.21\right.$ by PGSE, ${ }^{23} T^{*}$ $=1.50-2.17, \rho_{r} \geqslant 0.20$ by PGSE (Ref. 30)], krypton $\left(\varepsilon / k_{B}\right.$ $=178.9 \mathrm{~K} ; T^{*}=1.67, \rho_{r} \geqslant 0.028$ by tracer $\left.^{31}\right)$, carbon dioxide $\left(\varepsilon / k_{B}=195.2 \mathrm{~K} ; T^{*}=1.40-1.78, \rho_{r} \geqslant 0.036\right.$ by PGSE ${ }^{26} T^{*}$ $=1.40-1.91, \quad \rho_{r} \geqslant 0.0072$ by tracer, $^{32} T^{*}=1.40-1.78, \rho_{r}$ $\geqslant 0.010$ by $\left.\operatorname{tracer}^{33}\right)$, and ethylene $\left[\varepsilon / k_{B}=224.7 \mathrm{~K} ; T^{*}\right.$ $=1.33, \rho_{r} \geqslant 0.34$ by PGSE (Ref. 24)]. The lowest density of $0.0046 \mathrm{~g} \mathrm{~cm}^{-3}$ in the present work corresponds to $\rho_{r}$ $=0.0143$, which is much lower than those so far obtained by PGSE method and is comparable to those by tracer method. $^{30-33}$

When the density is lowered, the self-diffusion coefficient depends more strongly on the density; the self-diffusion coefficient diverges to the infinity in the zero-density limit. Thus the sample density needs to be determined very precisely in order to establish the density dependence of diffusion at low densities. The advantage of our sealed-tube method $^{1}$ is the separability of density and temperature effects. In this study, the density is determined in a high precision of $1 \%$ by making use of the proton chemical shift coupled with the PVT data of water. ${ }^{34}$ The precise density measurement has become possible owing to the high-field strength of the superconductor magnet $(11.3 \mathrm{~T}, 500 \mathrm{MHz}$ for $\left.{ }^{1} \mathrm{H}\right)$ and the high-level temperature homogeneity. The product $\rho D / \sqrt{T}$ is determined accurately enough to allow detailed comparison to theoretical analysis.

In the following section, we outline the simple gas kinetic theory for the self-diffusion coefficient. Section III contains the procedures for density measurement, diffusion measurement, and MD simulation. In Sec. IV, the temperature and density dependencies of the self-diffusion coefficient for supercritical water in the low-density limit are discussed in relation to the simple gas kinetic theory. Conclusions are given in Sec. V.

\section{THEORETICAL BACKGROUND}

We briefly review the gas kinetic theory for the selfdiffusion coefficient. ${ }^{2-4}$ Let us consider dilute gases in which the intermolecular distance is significantly larger than the molecular size. On the basis of the Chapman-Enskog solution to the Boltzmann equation, the self-diffusion coefficient is expressed as

$$
D=\frac{D_{\mathrm{HS}}}{\left\langle\Omega^{(1,1)}\right\rangle}=\frac{3}{8 \pi^{1 / 2}}\left(\frac{k_{B} T}{m}\right)^{1 / 2} \frac{1}{\rho_{n} \sigma^{2}\left\langle\Omega^{(1,1)}\right\rangle},
$$

where $D_{\mathrm{HS}}$ is the self-diffusion coefficient for dilute hard sphere gases, $k_{B}$ is the Boltzmann constant, $T$ is the temperature, $m$ is the mass, $\rho_{n}$ is the number density, $\sigma$ is the diameter, and $\left\langle\Omega^{(1,1)}\right\rangle$ is the collision integral, which is a function of the intermolecular potential and temperature. It should be noted that the density and temperature dependencies of $D_{\mathrm{HS}}$ are reduced to the constancy of $\rho D_{\mathrm{HS}} / \sqrt{T}$.

The integral $\left\langle\Omega^{(1,1)}\right\rangle$ has been rigorously obtained for the 6-12 LJ potential and is tabulated ${ }^{2}$ as a function of the scaled temperature $T^{*}=k_{B} T / \varepsilon$. When the temperature is increased, $\left\langle\Omega^{(1,1)}\right\rangle$ for LJ potential monotonically decreases as a result of the increasing effect of the kinetic energy against the attractive potential. Recently, an extensive MD study of the LJ potential has been performed over a wide range of density including the extremely low-density region $\left(\rho_{r} \geqslant 0.016\right){ }^{35}$ The $\rho D$ values thus obtained at extremely low densities are in agreement with those calculated by the Boltzmann equation with surprising accuracy. There has been an attempt to calculate $\left\langle\Omega^{(1,1)}\right\rangle$ for polar gases by using the simple Stockmayer (6-12-3) potential (Lennard-Jones potential with a point dipole) on the assumption that the relative orientation of dipoles does not change during a collision. ${ }^{36}$ The results are in good agreement with the experimental transport properties for various kinds of polar gases. For water, however, particular difficulties in the application have been mentioned in view of the high polarizability and the directional hydrogen bonding interactions. ${ }^{36}$

To the best of our knowledge, there has been no analytical work on the collision integral for water by explicitly taking into account the anisotropic intermolecular potential and the rotational degrees of freedom. Thus, we have performed the MD simulation to determine the $D$ values for super- and subcritical water in the extremely low-density region of $0.001-0.10 \mathrm{~g} \mathrm{~cm}^{-3}$. In the previous paper, ${ }^{1} \mathrm{MD}$ simulation of the TIP4P-FQ model well reproduced the diffusion coefficients for super- and subcritical water obtained by our NMR measurements. In computer simulation methods, significant progress in reproduction of experimental results has been achieved. ${ }^{37-43}$ Thus the overall tendency of the MD simulation results should be valuable. It should be noted that the TIP4P-FQ model excellently reproduced the NMR experimental results on the nonmonotonic density dependence of the rotational correlation time $\tau_{2 \mathrm{R}}$ for low- and medium-density supercritical water. ${ }^{44}$ 
For fluids at higher densities, a modification in the solution to the Boltzmann equation must be made since the effect of the finite molecular size must be taken into account. For the LJ fluids, the initial slope of the product $\rho D$ against $\rho$ has been calculated numerically from the approximate theory for the Boltzmann equation developed by Benett and Curtiss. ${ }^{45}$ The slope calculated is slightly negative. The slope obtained by the modified Boltzmann equation is in good agreement with the recent MD results. ${ }^{35}$ The hard sphere approximation called the Enskog theory has been widely used for dense gases because binary collisions can be clearly described for hard spheres even at high densities. In the Enskog theory, the enhancement in the collision frequency is introduced by multiplying $\rho_{n}$ by $g(\sigma)$, where $g(\sigma)$ is the radial distribution function at the contact distance $(\sigma)$ for hard spheres. ${ }^{46}$ The $g(\sigma)$ for hard spheres can be obtained by the CarnahanStarling expression and is $g(\sigma)=(1-0.5 \eta) /(1-\eta)^{3}$, where $\eta=(\pi / 6) \rho_{n} \sigma^{3} .^{47}$ The $g(\sigma)$ is unity in the zero-density limit and monotonically increases with increasing density, leading to the negative initial slope of $\rho D$ against $\rho .^{48}$ It is to be noted that this expression is based on the hard sphere approximation and the attractive interaction is neglected.

\section{PROCEDURES}

\section{A. Density measurements}

A precise density measurement is essential for the study on the density dependence of the self-diffusion of supercritical water in the low-density region. The sealed-tube method employed in this study is suitable for the purpose as previously referred to. ${ }^{1}$ In this method, water is sealed in a quartz tube and a supercritical state is realized by raising the temperature. In the preceding paper, ${ }^{1}$ we determined the density by measuring the mass of the sample water and the bore volume of the tube. The precision of the mass-volume method is $0.01-0.02 \mathrm{~g} \mathrm{~cm}^{-3}$, which is insufficient for the present study in the extremely low-density region. ${ }^{49}$ In this study, we have overcome the difficulty by taking advantage of the proton chemical shift. The proton chemical shift is a powerful probe as the $P V T$ relation of water is experimentally formulated at high temperatures under precise temperature regulation. ${ }^{34}$

The density in the one-phase region is uniquely determined in a quartz tube (closed system) by the transition temperature $T_{t}$ at which the boundary between the liquid and gas phases disappears. The density in a supercritical state is equal to that of the gas branch of the coexistence curve at $T_{t}$ when the density is lower than the critical. The PVT data for water are released by the International Association for the Properties of Water and Steam (IAPWS). ${ }^{34}$ The $T_{t}$ can be determined according to the temperature dependence of the proton chemical shift $\delta$ of the gas phase. In Fig. 1, the temperature dependence of the $\delta$ is shown for a sample at $0.0046 \mathrm{~g} \mathrm{~cm}^{-3}$ in the vicinity of $T_{t}$. As can be seen, $\delta$ has a maximum. The temperature of the maximum $\delta$ is $T_{t}$ because the temperature dependence of the hydrogen-bond strength, which is in positive correlation with $\delta$, turns over at $T_{t}$ in a closed system. Below $T_{t}$, the signal comes from the gas branch of the coexistence curve ${ }^{50}$ and the hydrogen bonding

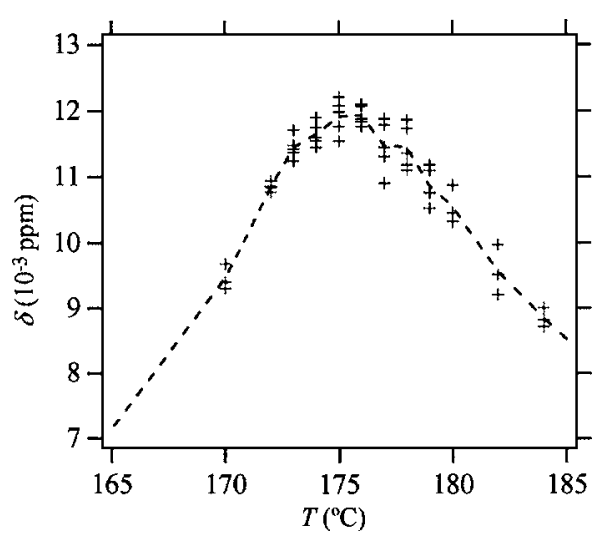

FIG. 1. The chemical shift $\delta$ plotted against temperature for the sample at the lowest density of $0.0046 \mathrm{~g} \mathrm{~cm}^{-3}$. The $\delta$ for the gas branch of the liquidgas coexistence curve at $150{ }^{\circ} \mathrm{C}\left(0.0025 \mathrm{~g} \mathrm{~cm}^{-3}\right)$ is set to zero. The dashed line indicates the average of the repetitions.

in the gas phase enhances with increasing temperature due to the increase in the density. ${ }^{51}$ Above $T_{t}$, the density of the encapsulated water is constant and the hydrogen-bond strength reduces monotonically with increasing temperature due to the increase in the kinetic energy of water molecules. The ${ }^{1} \mathrm{H}$ spectra were taken at temperature intervals of $1{ }^{\circ} \mathrm{C}$ near $T_{t}$ in a fully automated manner. At each temperature, the ${ }^{1} \mathrm{H}$ spectra were taken five times consecutively with $10 \mathrm{~min}$ intervals after the temperature equilibration (within $10 \mathrm{~min}$ ). The effect of supersaturation or superheating is negligible at high temperatures and low densities. This was confirmed by changing the temperature both upward and downward and the $T_{t}$ values thus determined were almost identical. For the samples at $0.0425 \mathrm{~g} \mathrm{~cm}^{-3}$ and below, gaseous acetone was used as an external reference. ${ }^{52} T_{t}$ and the density could be then determined in a precision of $\pm 1{ }^{\circ} \mathrm{C}$ and $\pm 1 \%$, respectively. In this study, we set the temperature homogeneity in the sample much higher (within 0.5 and $2{ }^{\circ} \mathrm{C}$ at 200 and $400{ }^{\circ} \mathrm{C}$, respectively) than that in Ref. 1 because of the absence of the convection effect in the low-density region.

The sample was prepared as follows. Water was purified using a Milli-Q Labo (Millipore) filter system. Quartz sample tubes $[1.5 \mathrm{~mm}$ inner diameter (i.d.) and $2.7 \mathrm{~mm}$ outer diameter (o.d.)] were used for the diffusion measurements. The procedure for confining water into the quartz tube is the same as that described in the previous paper (see Fig. 2 in Ref. 1) except for the following: the air inside was once exchanged by argon to remove paramagnetic oxygen and then the argon was selectively removed by a vacuum pump from the sample frozen with $\mathrm{NaCl}$-containing ice. ${ }^{53}$ This procedure is needed because of the extremely low density in the present study. The solubility of quartz in hot water is so small $^{54}$ that it must have little effect on $T_{t}$.

\section{B. Diffusion measurements}

Diffusion measurement techniques and the apparatus have been described in detail in the previous paper. ${ }^{1}$ Only important aspects are briefly described here. Hightemperature experiments were possible by setting a hightemperature probe ${ }^{1}$ in a NMR spectrometer (JEOL ECA500 wide-bore type) with a superconductor magnet $(11.3 \mathrm{~T})$. The 
intensity of the half-sine-shaped magnetic field gradient $g$ was fixed at $0.671 \mathrm{mT} \mathrm{cm}^{-1}$. In each measurement, we took ten different values of the duration $\delta$ of the magnetic field gradient pulse. To check the reproducibility, the measurement was repeated five times for each sample at each temperature. The uncertainty of $D$ was $\pm 1 \%$ among five runs consecutively performed for one sample. The total uncertainty of $D$ was estimated at about $\pm 5 \%$ by taking into account the error due to the sample resetting. The free induction decay (FID) signals were accumulated 1-32 times for each $\delta$. The signal-to-noise ratio for $\delta=0 \mathrm{~ms}$ exceeded 150 even at the lowest density of $\rho=0.0046 \mathrm{~g} \mathrm{~cm}^{-3}$.

\section{Molecular dynamics simulation}

The simulation procedure was the same as that described in Ref. 44. The TIP4P-FQ model was adopted as the potential function. ${ }^{55}$ The molecular dynamics simulation was performed using a time-reversible quarternion algorithm. ${ }^{56}$ The molecular dynamics was carried out for $2.5 \mathrm{~ns}$ at densities of $0.005 \mathrm{~g} \mathrm{~cm}^{-3}$ and higher and for $7.5 \mathrm{~ns}$ at densities of $0.002 \mathrm{~g} \mathrm{~cm}^{-3}$ and lower, with a time step of $1.0 \mathrm{fs}$. A long simulation is required toward low density because the velocity autocorrelation time becomes large in the dilute region. It is to be noted that, owing to the definition of the fluctuating charge, the TIP4P-FQ model can incorporate the variation of the dipole moment of the water molecule in response to the surrounding environment. The dipole moment, which is a function of the thermodynamic condition, is the most important parameter for the MD of water.

\section{RESULTS AND DISCUSSION}

The density and temperature effects can be separately investigated in supercritical water whose density is continuously varied at each temperature fixed. Thus we can scrutinize the kinetic and potential effects in a separate manner. We have examined the density effect on the self-diffusion of water over a density range of $0.0046-0.0650 \mathrm{~g} \mathrm{~cm}^{-3}$ at fixed temperatures in the range of $200-400{ }^{\circ} \mathrm{C}$ and the temperature effect on the isochore in the same temperature range. The self-diffusion coefficients determined are summarized in Table I. We discuss the self-diffusion for supercritical water in the zero-density limit and the temperature and density effects at low densities.

\section{A. Self-diffusion in the zero-density limit}

In this section, we examine the self-diffusion coefficient for water in the zero-density limit. The self-diffusion coefficient $D$ obtained for water at extremely low densities is shown in Fig. 2 in the form of $\rho D / \sqrt{T}$.

First we confirm the reliability of the present experimental results. The self-diffusion coefficient $D$ is very strongly dependent on the density in the low-density region as shown in Table Ia and the accuracy of the $\rho D / \sqrt{T}$ value is controlled by the uncertainty of the density; the accuracy of the absolute value of $D$ is much better. We have checked the reliability of the density measurement procedure newly developed in this study by examining whether the $\rho D / \sqrt{T}$ value is con-
TABLE I. The self-diffusion coefficients for water determined by (a) NMR experiment and (b) MD simulation in high-temperature and low-density conditions including supercritical.

\begin{tabular}{|c|c|c|c|}
\hline (a) & $T\left({ }^{\circ} \mathrm{C}\right)$ & $\rho\left(\mathrm{g} \mathrm{cm}^{-3}\right)$ & $D\left(10^{-6} \mathrm{~m}^{2} \mathrm{~s}^{-1}\right)^{\mathrm{a}}$ \\
\hline & 200 & $0.0046 \pm 0.0002$ & 4.87 \\
\hline & & $0.0079 \pm 0.0002^{\mathrm{a}}$ & 2.89 \\
\hline & 300 & $0.0046 \pm 0.0002$ & 6.61 \\
\hline & & $0.0121 \pm 0.0002$ & 2.57 \\
\hline & & $0.0140 \pm 0.0003$ & 2.09 \\
\hline & & $0.0159 \pm 0.0004$ & 1.93 \\
\hline & & $0.0321 \pm 0.0004$ & 0.949 \\
\hline & & $0.0332 \pm 0.0004$ & 0.867 \\
\hline & & $0.0337 \pm 0.0004$ & 0.914 \\
\hline & & $0.0425 \pm 0.0004$ & 0.702 \\
\hline & & $0.0432 \pm 0.0004$ & 0.696 \\
\hline & & $0.0462 \pm 0.0005^{\mathrm{b}}$ & 0.637 \\
\hline & 400 & $0.0046 \pm 0.0002$ & 8.04 \\
\hline & & $0.0121 \pm 0.0002$ & 3.18 \\
\hline & & $0.0140 \pm 0.0003$ & 2.63 \\
\hline & & $0.0159 \pm 0.0003$ & 2.37 \\
\hline & & $0.0321 \pm 0.0005$ & 1.19 \\
\hline & & $0.0332 \pm 0.0005$ & 1.10 \\
\hline & & $0.0337 \pm 0.0005$ & 1.14 \\
\hline & & $0.0425 \pm 0.0007$ & 0.880 \\
\hline & & $0.0432 \pm 0.0007$ & 0.885 \\
\hline & & $0.0584 \pm 0.0010$ & 0.648 \\
\hline & & $0.0625 \pm 0.0011$ & 0.593 \\
\hline & & $0.0650 \pm 0.0011$ & 0.585 \\
\hline \multirow[t]{17}{*}{ (b) } & $T\left({ }^{\circ} \mathrm{C}\right)$ & $\rho\left(\mathrm{g} \mathrm{cm}^{-3}\right)$ & $D\left(10^{-6} \mathrm{~m}^{2} \mathrm{~s}^{-1}\right)^{\mathrm{c}}$ \\
\hline & 200 & 0.001 & $23.1 \pm 1.5$ \\
\hline & & 0.002 & $10.9 \pm 0.6$ \\
\hline & & 0.005 & $4.43 \pm 0.20$ \\
\hline & & 0.01 & $2.19 \pm 0.06$ \\
\hline & 300 & 0.001 & $29.05 \pm 2.18$ \\
\hline & & 0.002 & $13.56 \pm 0.41$ \\
\hline & & 0.005 & $5.40 \pm 0.12$ \\
\hline & & 0.01 & $2.82 \pm 0.09$ \\
\hline & & 0.05 & $0.552 \pm 0.023$ \\
\hline & & 0.10 & $0.273 \pm 0.004$ \\
\hline & 400 & 0.001 & $34.76 \pm 1.05$ \\
\hline & & 0.002 & $17.36 \pm 0.30$ \\
\hline & & 0.005 & $6.72 \pm 0.22$ \\
\hline & & 0.01 & $3.40 \pm 0.15$ \\
\hline & & 0.05 & $0.664 \pm 0.013$ \\
\hline & & 0.10 & $0.338 \pm 0.007$ \\
\hline
\end{tabular}

${ }^{\mathrm{a}}$ The error is $\pm 5 \%$.

${ }^{\mathrm{b}}$ The gas branch of the liquid-gas coexistence curve. The error in density is due to the temperature instability of $\pm 0.5^{\circ} \mathrm{C}$.

${ }^{c}$ The error is expressed at $95 \%$ confidence level.

sistent with that on the gas branch of the liquid-gas coexistence curve, where the vapor density is uniquely determined by the temperature. In Fig. 2 , it is seen that the $\rho D / \sqrt{T}$ values at 200 and $300{ }^{\circ} \mathrm{C}$ are in harmony with the values on the coexistence curve. This fact supports the reliability of the density measurement based on the proton chemical shift. The $D$ value obtained by the present MD simulation is listed in Table Ib. The MD simulation well reproduces the magnitude 


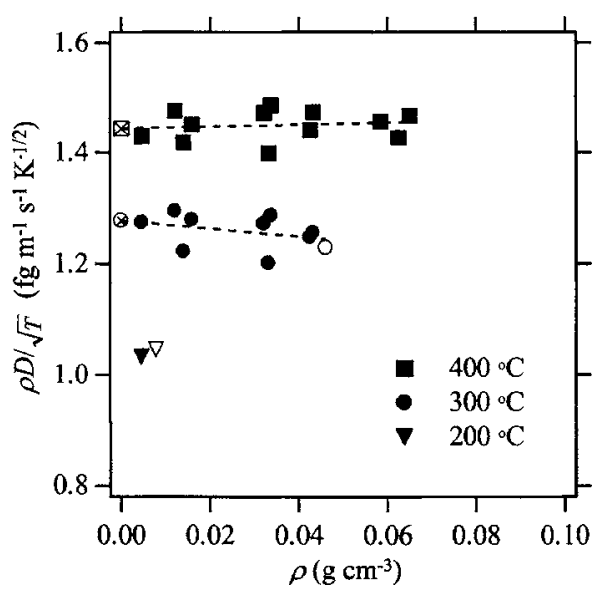

FIG. 2. The product of the self-diffusion coefficients of water and the density divided by square root of the temperature, $\rho D / \sqrt{T}$, plotted against the density at 200,300 , and $400{ }^{\circ} \mathrm{C}$. The open symbols indicate the values on the gas branch of the liquid-gas coexistence curve. The dashed line is linearly fitted to the experimental values and the crossed symbols indicate the values extrapolated to the zero-density limit, $(\rho D)^{0} / \sqrt{T}$. At $200{ }^{\circ} \mathrm{C}$, the $\rho D / \sqrt{T}$ value at the lowest density of $0.0046 \mathrm{~g} \mathrm{~cm}^{-3}$ is used as the $(\rho D)^{0} / \sqrt{T}$ value because the steam-density region is too narrow to allow the linear fit of $\rho D / \sqrt{T}$ against $\rho$.

of the present experimental $D$ values. The agreement is also satisfactory with the results of the MD simulations by Yoshii et al. using a polarizable potential model proposed by Dang (RPOL model) ${ }^{38}$ at higher densities $\left(\geqslant 0.027 \mathrm{~g} \mathrm{~cm}^{-3}\right) .39$

The extrapolation of the experimental values at 200, 300, and $400{ }^{\circ} \mathrm{C}$ to the zero-density limit gives the $\rho D / \sqrt{T}$ values of $1.03,1.28$, and $1.44 \mathrm{fg} \mathrm{m}^{-1} \mathrm{~s}^{-1} \mathrm{~K}^{-1 / 2}$ (f, femto), respectively. In order to see the effect of attractive interactions, we compare $\rho D / \sqrt{T}$ in the zero-density limit, denoted as $(\rho D)^{0} / \sqrt{T}$, with those predicted by the gas kinetic theory and MD simulation in the following section.

\section{B. Temperature dependence of the self-diffusion}

In this section, we discuss the temperature effect on the self-diffusion coefficient for supercritical water in the zerodensity limit. In Fig. 3, $(\rho D)^{0} / \sqrt{T}$ is plotted against the tem-

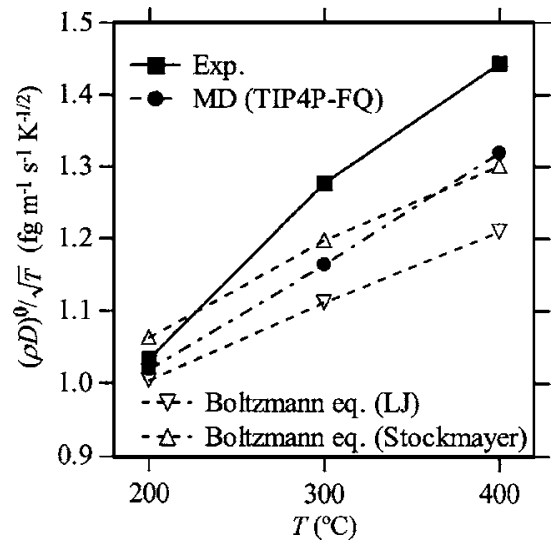

FIG. 3. The product $(\rho D)^{0} / \sqrt{T}$ for water plotted against temperature. The values calculated by the Chapman-Enskog solution of the Boltzmann equation by using the LJ potential and the Stockmayer potential are taken from Refs. 2 and 36, respectively. perature. It is seen that $(\rho D)^{0} / \sqrt{T}$ decreases with decreasing temperature. This indicates that the dynamic effect of the strong attractive interactions between water molecules is stronger at lower temperatures. The overall tendency of the temperature dependence of $(\rho D)^{0} / \sqrt{T}$ is well reproduced by MD simulation, as shown in Fig. 3. The $(\rho D)^{0} / \sqrt{T}$ values in Fig. 3 are significantly smaller than that of the hard sphere model, $\left(\rho D_{\mathrm{HS}}\right)^{0} / \sqrt{T}$, which is $1.95 \mathrm{fg} \mathrm{m}^{-1} \mathrm{~s}^{-1} \mathrm{~K}^{-1 / 2}$ with $\sigma$ at $2.64 \AA .{ }^{19,57}$ The large difference can be accounted for by considering the presence of scattering or orbiting of an encounter molecule in the attractive part of the potential.

Let us test to what extent the collision integral calculated for the LJ potential or the Stockmayer (point-dipole) potential can explain the self-diffusion for low-density water. The $(\rho D)^{0} / \sqrt{T}$ values calculated by using the collision-integral values for LJ (Refs. 2 and 19) and Stockmayer ${ }^{36}$ potentials are included in Fig. 3 for comparison. They are in fairly good agreement despite the marked simplification of intermolecular potentials. This is probably because the experimental properties of water are used to determine the potential parameters, experimental virial coefficients for LJ potential and viscosity data for Stockmayer potential. MD simulation with the LJ potential also well reproduces the temperature dependence of $(\rho D)^{0} / \sqrt{T} .^{35}$ In this context, LJ or Stockmayer approximations can provide a rough value of $D$ for practical use. It should be noted, however, that higher approximations like TIP4P-FQ model are desired to be used for a better understanding of the detailed mechanism of the collision and orbiting in supercritical water dynamics.

The temperature dependence of $D$ can be separated into the kinetic (hard sphere) and the (attractive) potential contributions. The activation energy for the self-diffusion at constant density can be decomposed into

$$
E_{D}=-k_{B}\left(\frac{\partial \ln D}{\partial(1 / T)}\right)_{\rho}=E_{\mathrm{pot}}+E_{\mathrm{kin}},
$$

where $E_{\mathrm{pot}}$ is the potential contribution and the kinetic contribution $E_{\mathrm{kin}}$ is introduced as

$$
E_{\mathrm{kin}}=-k_{B}\left(\frac{\partial \ln D_{\mathrm{HS}}}{\partial(1 / T)}\right)_{\rho}=k_{B} T .
$$

The $E_{D}$ is $7 \pm 1 \mathrm{~kJ} \mathrm{~mol}^{-1}$ in the thermodynamic conditions studied. The kinetic contribution $E_{\text {kin }}$ is $2.6 \mathrm{~kJ} \mathrm{~mol}^{-1}, 58$ and thus the contribution of the potential effect $E_{\text {pot }}$ is 4-5 kJ mol${ }^{-1}$. The $E_{\text {pot }}$ value is one-half to one-fourth of the hydrogen-bond dissociation free energy $E_{\delta}$ obtained from the proton chemical shift at $400{ }^{\circ} \mathrm{C} ; E_{\delta}$ is $18 \pm 11 \mathrm{~kJ} \mathrm{~mol}^{-1}$ at $0.19 \mathrm{~g} \mathrm{~cm}^{-3}$ and is expected not to be smaller at a lower density. ${ }^{20}$ This can be understood as follows. $E_{D}$ corresponds to the energy to break the hydrogen bonding to diffuse. $E_{\delta}$ is, on the other hand, related to the equilibrium free energy change when a specific pair of water molecules is brought from a hydrogen bonding configuration to an infinite separation. The pair of water in the "activated state for diffusion" are on the way to an infinite separation and thus $E_{D}$ is expected to be somewhat smaller than the static $E_{\delta}$.

The temperature dependence of $\rho D$ is often approximated empirically by a power of the temperature: 
TABLE II. The local density $\rho_{l}$ calculated by the MD simulation of TIP4P-FQ model.

\begin{tabular}{ll}
\hline \hline$\rho\left(\mathrm{g} \mathrm{cm}^{-3}\right)$ & $\rho_{l} / \rho$ \\
\hline 0.40 & 1.10 \\
0.20 & 1.21 \\
0.10 & 1.28 \\
0.005 & 1.34 \\
0.001 & 1.37 \\
\hline \hline
\end{tabular}

$\rho D \propto T^{B} .^{18,22}$ The $B$ value for $(\rho D)^{0}$ is 1.4 and 1.2 for the present experiment and MD simulation with TIP4P-FQ model, respectively. Previously it is reported that the $B$ value for water is obtained as $0.76 ;{ }^{18}$ note that this is obtained at higher temperatures $\left(400-700{ }^{\circ} \mathrm{C}\right)$ and densities $\left(0.10-0.60 \mathrm{~g} \mathrm{~cm}^{-3}\right)$. The difference in the $B$ values between the present and previous studies can arise from the strong attractive potential effect at the low densities and temperatures. The present $B$ value is a largest among the values reported for various substances; the $B$ value is ranged from 0.75 to 1.1 for less polar substances. ${ }^{18,22}$

\section{Density dependence of the self-diffusion at low densities}

In this section, we examine the density dependence of the self-diffusion coefficient at extremely low densities. As seen in Fig. 2, the initial slope of $\rho D / \sqrt{T}$ against $\rho$ is almost zero at $400{ }^{\circ} \mathrm{C}$ and slightly negative at $300{ }^{\circ} \mathrm{C}$. The magnitude of the slope and its temperature dependence are well reproduced by the MD simulation of TIP4P-FQ model as inferred in Table Ib. ${ }^{59}$ MD simulation by the LJ potential ${ }^{35}$ gives similar slope values. ${ }^{60}$ The experimental initial slopes for other substances are, in general, slightly negative or almost zero. ${ }^{22-26,30-33}$ On the other hand, the Enskog theory for hard spheres at higher densities gives a much more negative slope than the present. ${ }^{3}$

Here we test whether the density dependence of $\rho D / \sqrt{T}$ can be accounted for or not in analogy with the Enskog theory in terms of $g(\sigma)$. The $g(\sigma)$ for water [the intermolecular site-site radial distribution functions $g_{\mathrm{OO}}(r), g_{\mathrm{OH}}(r)$, and $\left.g_{\mathrm{HH}}(r)\right]$ needs to be obtained from other experiments (difficult at low densities) or computer simulations. In this study, we obtained $g(\sigma)$ by MD simulation. Since $g(\sigma)$ at contact cannot be clearly defined for water, we use the density in the first coordination shell divided by the bulk density, $\rho_{l} / \rho$, obtained by integrating the oxygen-oxygen radial distribution function $g_{\mathrm{OO}}(r)$ from $2.4 \AA$ up to the first minimum of $5.0 \AA .{ }^{61}$ The $\rho_{l} / \rho$ values at $400{ }^{\circ} \mathrm{C}$ is listed in Table II. The $\rho_{l} / \rho$ values increase with decreasing density in accordance with the previous studies at higher densities. ${ }^{20,21,62-64}$ The slope of $\rho D_{\mathrm{HS}} / \sqrt{T}\left(\rho_{l} / \rho\right)$ against $\rho$ is positive, in disagreement with the present experimental results. Thus the density dependence of the self-diffusion for water cannot be explained in terms of the Enskog theory, where $\rho$ is multiplied by $g(\sigma)$ to enhance the collision frequency. ${ }^{65,66}$

\section{CONCLUSIONS}

Using PGSE method with a precision high-temperature NMR probe, we have determined the self-diffusion coefficient $D$ for water in the low-density region of $0.0046-$ $0.0079,0.0046-0.0462$, and $0.0046-0.0650 \mathrm{~g} \mathrm{~cm}^{-3}$ at 200 , 300 , and $400{ }^{\circ} \mathrm{C}$, respectively. The water density in the extremely low-density region is successfully determined by the method newly developed on the basis of the density dependence of the proton chemical shift. Let us summarize the present results.

(1) The product $(\rho D)^{0} / \sqrt{T}$ obtained by the extrapolation to the zero-density limit is significantly smaller than the one in the hard sphere model.

(2) The $D$ values obtained by the experiment are in agreement with those obtained by the MD simulation with TIP4P-FQ model.

(3) The product $(\rho D)^{0} / \sqrt{T}$ in the zero-density limit is smaller at a lower temperature.

(4) The slope of $\rho D / \sqrt{T}$ against $\rho$ is almost zero at $400{ }^{\circ} \mathrm{C}$ and slightly negative at $300{ }^{\circ} \mathrm{C}$.

The absolute value of $(\rho D)^{0} / \sqrt{T}$ (1) reflects the strong attractive interactions in the extremely low-density region. The temperature dependence of $(\rho D)^{0} / \sqrt{T}$ (2) indicates an increasing effect of attractive interactions at a lower temperature. The tendency in the temperature dependence is also well reproduced by MD simulation. The density dependence (3) is consistent with the density dependence of $\rho D / \sqrt{T}$ by MD simulation. The fair agreement (4) has encouraged us to perform a detailed analysis by using MD simulation to elucidate the diffusion mechanism controlled by scattering or orbiting in a strongly attractive part of potential. Also a mixture system is desired to be studied to understand intermolecular interactions between similar and dissimilar molecules from a dynamical viewpoint. ${ }^{53}$

\section{ACKNOWLEDGMENT}

This work was supported by the Next Generation Super Computing Project, Nanoscience Program, MEXT, Japan.

${ }^{1}$ K. Yoshida, C. Wakai, N. Matubayasi, and M. Nakahara, J. Chem. Phys. 123, 164506 (2005).

${ }^{2}$ J. O. Hirschfelder, C. F. Curtiss, and R. B. Bird, Molecular Theory of Gases and Liquids (Wiley, New York, 1954).

${ }^{3}$ S. Chapman and T. G. Cowling, The Mathematical Theory of NonUniform Gases, 3rd ed. (Cambridge University Press, London, 1970).

${ }^{4}$ D. A. McQuarrie, Statistical Mechanics (Harper \& Row, New York, 1976), Chap. 19.

${ }^{5}$ Water, A Comprehensive Treatise, edited by F. Franks (Plenum, New York, 1972), Vols. 1-7.

${ }^{6}$ J. S. Seewald, Nature (London) 370, 285 (1994).

${ }^{7}$ P. A. Marrone, T. A. Arias, W. A. Peters, and J. W. Tester, J. Phys. Chem. A 102, 7013 (1998).

${ }^{8}$ N. Akiya and P. E. Savage, Chem. Rev. (Washington, D.C.) 102, 2725 (2002); J. T. Henrikson, C. R. Grice, and P. E. Savage, J. Phys. Chem. A 110, 3627 (2006).

${ }^{9}$ J. M. DeSimone, Science 297, 799 (2002).

${ }^{10}$ D. Miksa, J. Li, and T. B. Brill, J. Phys. Chem. B 106, 11107 (2002).

${ }^{11}$ K. Yoshida, C. Wakai, N. Matubayasi, and M. Nakahara, J. Phys. Chem. A 108, 7479 (2004).

${ }^{12}$ Y. Nagai, N. Matubayasi, and M. Nakahara, J. Phys. Chem. A 109, 3550 (2005); 109, 3558 (2005). 
${ }^{13}$ N. Matubayasi and M. Nakahara, J. Chem. Phys. 122, 074509 (2005).

${ }^{14}$ S. Morooka, C. Wakai, N. Matubayasi, and M. Nakahara, J. Phys. Chem. A 109, 6610 (2005).

${ }^{15}$ H. Weingärtner and E. U. Franck, Angew. Chem., Int. Ed. 44, 2672 (2005).

${ }^{16}$ A. H. Harvey and R. D. Mountain, Ind. Eng. Chem. Res. 42, 404 (2003).

${ }^{17}$ M. Nakahara, Proceedings of the 14th International Conference on the Properties of Water and Steam, Kyoto, 2004, edited by M. Nakahara, N. Matubayasi, M. Ueno, K. Yasuoka, and K. Watanabe (Maruzen Co., Ltd., Tokyo, 2005), p. 12.

${ }^{18}$ W. J. Wilber, G. A. Hoffman, and J. Jonas, J. Chem. Phys. 74, 6875 (1981).

${ }^{19}$ R. C. Reid, J. M. Prausnitz, and B. E. Poling, The Properties of Gases and Liquids, 4th ed. (McGraw-Hill, New York, 1987).

${ }^{20}$ N. Matubayasi, C. Wakai, and M. Nakahara, J. Chem. Phys. 107, 9133 (1997).

${ }^{21}$ N. Matubayasi, C. Wakai, and M. Nakahara, J. Chem. Phys. 110, 8000 (1999).

${ }^{22}$ P. W. E. Peereboom, H. Luigjes, and K. O. Prins, Physica A 156, 260 (1989).

${ }^{23}$ P. H. Oosting and N. J. Trappeniers, Physica (Amsterdam) 51, 418 (1971).

${ }^{24}$ B. Arends, K. O. Prins, and N. J. Trappeniers, Physica A 107, 307 (1981).

${ }^{25}$ F. Khoury and R. Kobayashi, J. Chem. Phys. 55, 2439 (1971).

${ }^{26}$ P. Etesse, J. A. Zega, and R. Kobayashi, J. Chem. Phys. 97, 2022 (1992).

${ }^{27}$ E. S. Baker, D. R. Brown, and J. Jonas, J. Phys. Chem. 88, 5425 (1984).

${ }^{28}$ N. Asahi and Y. Nakamura, Ber. Bunsenges. Phys. Chem. 101, 831 (1997).

${ }^{29}$ N. Asahi and Y. Nakamura, J. Chem. Phys. 109, 9879 (1998).

${ }^{30}$ K. R. Harris, Physica A 94, 448 (1978).

${ }^{31}$ N. J. Trappeniers and J. P. J. Michels, Chem. Phys. Lett. 18, 1 (1973).

${ }^{32}$ H. A. O'Hern and J. J. Martin, Ind. Eng. Chem. 47, 2081 (1955).

${ }^{33}$ S. Takahashi and H. Iwasaki, Bull. Chem. Soc. Jpn. 39, 2105 (1966).

${ }^{34}$ Release on the IAPWS Formulation 1995 for the Thermodynamic Properties of Ordinary Water Substances for General and Scientific Use, URL: http://www.iapws.org

${ }^{35}$ K. Meier, A. Laesecke, and S. Kabelac, J. Chem. Phys. 121, 9526 (2004). The simulation data are available in the electric archive of the NIST Physical and Chemical Properties Division, ftp:// FTP.Boulder.NIST.Gov/pub/fluids/Lennard-Jones

${ }^{36}$ L. Monchick and E. A. Mason, J. Chem. Phys. 35, 1676 (1961).

${ }^{37}$ H. J. C. Berendsen, J. R. Grigera, and T. P. Straatsma, J. Phys. Chem. 91, 6269 (1987)

${ }^{38}$ L. X. Dang, J. Chem. Phys. 97, 2659 (1992).

${ }^{39}$ N. Yoshii, H. Yoshie, S. Miura, and S. Okazaki, J. Chem. Phys. 109, 4873 (1998).

${ }^{40}$ P. B. Balbuena, K. P. Johnston, P. J. Rossky, and J. K. Hyun, J. Phys. Chem. B 102, 3806 (1998).

${ }^{41}$ A. A. Chivalo and P. T. Cummings, Adv. Chem. Phys. 109, 115 (1999).

${ }^{42}$ M. S. Skaf and D. Laria, J. Chem. Phys. 113, 3499 (2000).

${ }^{43}$ C.-N. Yang and H. J. Kim, J. Chem. Phys. 113, 6025 (2000).

${ }^{44}$ N. Matubayasi, N. Nakao, and M. Nakahara, J. Chem. Phys. 114, 4107 (2000).

${ }^{45}$ D. E. Bennett and C. F. Curtiss, J. Chem. Phys. 51, 2811 (1969).

${ }^{46}$ J.-P. Hansen and I. R. McDonald, Theory of Simple Liquids (Harper \& Row, New York, 1976), Chap. 7.

${ }^{47}$ N. F. Carnahan and K. E. Starling, J. Chem. Phys. 51, 635 (1969).

${ }^{48}$ In more simple approximation, the slope of the product $\rho_{n} D$ against $\rho_{n}$ is also negative; $-(5 / 12) \pi \sigma^{3}$ as in Ref. 2.

${ }^{49}$ The precision is found to be less than that estimated in Ref. 1 for an unknown reason when it is tested in the extremely low-density region.

${ }^{50}$ The influence of the liquid phase is negligible in this setup below $T_{t}$ for the following reason. The spectra of the gas phase and liquid phase are separated in the chemical shift by 1-4 ppm (Ref. 20), which is much larger than the signal width. Furthermore, since the shim current was optimized for the gas phase, the liquid-phase spectrum does not appear.

${ }^{51}$ The magnetic susceptibility correction is not necessary in the present study since the present purpose is only to determine the $T_{t}$ and the chemical shift measurement is performed only in the vicinity of the $T_{t}$.

${ }^{52}$ An external reference is necessary for samples at low densities because the change in $\delta\left({ }^{1} \mathrm{H}\right)$ is smaller at lower temperatures. The acetone density was set to an order of magnitude smaller than the water density so that acetone is in the one-phase region in the vicinity of $T_{t}$ for water. Thus the density and magnetic susceptibility of acetone does not change in the vicinity of $T_{t}$ for water. Indeed, the change in $\delta\left({ }^{1} \mathrm{H}\right)$ for acetone was negligibly small compared with that for water.

${ }^{53}$ We have performed another independent experiment to see the effect of argon. When we did not remove argon by pumping, we observed that $\rho D / \sqrt{T}$ is significantly smaller than that shown in Fig. 3 when the water density was comparable to the argon density. The $D$ value for water in argon-water mixture is controlled by the water-water and water-argon intermolecular interactions and densities of both water and argon. When the water density is lowered to the zero-density limit with a constant atmospheric density of argon, $D$ for water approaches to a finite value (for infinitely dilute water in argon) and thus the product $\rho D / \sqrt{T}$ for water will be significantly smaller. When the water density is smaller than the argon density $(\sim 40 \mathrm{mM})$, the water diffusion is expected to be controlled dominantly by the water-argon interactions. In such conditions, however, the water density is below the detection limit. In order to study the dynamic intermolecular interactions over wide ranges of density and composition ratio, it is of great interest to investigate binary mixture systems of water and some other substances for which the self-diffusion coefficients can be determined with the densities controlled.

${ }^{54}$ C. E. Manning, Geochim. Cosmochim. Acta 58, 4831 (1994).

${ }^{55}$ S. W. Rick, S. J. Stuart, and B. J. Berne, J. Chem. Phys. 101, 6141 (1994).

${ }^{56}$ N. Matubayasi and M. Nakahara, J. Chem. Phys. 110, 3291 (1999).

${ }^{57} \mathrm{We}$ have fixed the hard sphere diameter in order to make the discussion clearer. The discussion in Sec. IV B would not be affected even if the hard sphere diameter were treated as a temperature-variable parameter because the temperature dependence of HS diameter is generally less than $10 \%$ in the temperature range in this study.

${ }^{58} \mathrm{The}$ value of $2.6 \mathrm{~kJ} \mathrm{~mol}^{-1}$ for $E_{\mathrm{kin}}$ is obtained at $350{ }^{\circ} \mathrm{C}$ since the data at 300 and 400 are used for the determination of $E_{D}$.

${ }^{59}$ The experimental values of the slope are $-0.8 \pm 0.7$ and $0.2 \pm 0.4 \mathrm{zg} \mathrm{m}^{2} \mathrm{~s}^{-1} \mathrm{~K}^{-1 / 2}\left(\mathrm{z}\right.$, zepto; $\left.10^{-21}\right)$ at 300 and $400^{\circ} \mathrm{C}$, respectively. The slope values by the MD simulation of TIP4P-FQ model are $-4 \pm 4$, $-0.2 \pm 0.4$, and $-0.3 \pm 0.3 \mathrm{zg} \mathrm{m}^{2} \mathrm{~s}^{-1} \mathrm{~K}^{-1 / 2}$ at 200,300 , and $400{ }^{\circ} \mathrm{C}$, respectively.

${ }^{60}$ The slope values in Ref. 35 are $-3.0,-1.3$, and $-0.92 \mathrm{zg} \mathrm{m}^{2} \mathrm{~s}^{-1} \mathrm{~K}^{-1 / 2}$ at 293,455 , and $617^{\circ} \mathrm{C}$, respectively. The slope values of the modified Boltzmann equation (Ref. 45) is one order of magnitude smaller (much more negative) than the present values and those in Ref. 35. The discrepancy has already been pointed out in Ref. 35 .

${ }^{61}$ Here we used $g_{\text {Oo }}(r)$ instead of the oxygen-hydrogen radial distribution function $g_{\mathrm{OH}}(r)$, which is used to calculate the number of hydrogen bonds, because the characteristic first peak of $g_{\mathrm{OH}}(r)$ is too weak to quantify in the extremely low-density region. The overall tendency described in Sec. IV C is not affected when the $g_{\mathrm{OO}}(r)$ is replaced by $g_{\mathrm{OH}}(r)$.

${ }^{62}$ L. W. Flanagin, P. B. Balbuena, K. P. Johnston, and P. J. Rossky, J. Phys. Chem. 99, 5196 (1995).

${ }^{63}$ R. D. Mountain, J. Chem. Phys. 110, 2109 (1999).

${ }^{64}$ B. Guillot and Y. Guissani, J. Chem. Phys. 114, 6720 (2001).

${ }^{65}$ The same comment can be made on the Enskog approach to the LJ fluid. While the initial slopes of $\rho D / \sqrt{T}$ against $\rho$ for the LJ fluids are negative in Refs. 35 and 45, the maximum value of $g(r)$ for the LJ fluid in Ref. 66 increases with decreasing density.

${ }^{66}$ R. D. Mountain, NIST Report No. NISTIR 6028, 1997 (unpublished). 